\title{
The child with urinary tract infection: a dilemma for the paediatrician
}

\author{
Chandra Abeysekera ${ }^{1}$ \\ Sri Lanka Journal of Child Health, 2005; 34: 30-43
}

(Key words: urinary tract infection, children)

Professor C C de Silva was born on the 25th of February 1904. He received his primary and secondary education at St Thomas College, Mount Lavinia. After one year at the Ceylon Medical College, he proceeded to the United Kingdom to complete his medical degree. He wasted no time in passing the MRCP and MD examinations. He later settled down to a general practice in Colombo. In 1949 he was appointed to the newly created Chair of Paediatrics of the then University of Ceylon. He accepted the challenge of organizing and developing the new Department of Paediatrics from its inception. He pioneered and organized undergraduate teaching in Sri Lanka. He was also responsible for the introduction of post-graduate training and the initiation of a DCH programme.

After the second Medical School at Peradeniya was started in 1964, he took up the challenge of organizing the Department of Paediatrics in the new medical school. He selected Herbert Aponso as the Senior Lecturer in Charge and continued to make weekly visits to Peradeniya until the department was firmly established. Professor C C retired prematurely in 1965 to take up an appointment abroad. In 1983, he was awarded the Doctorate of Science by the University of Peradeniya. His contributions to paediatrics in Sri Lanka are vast. It can be truly said that it was Prof. C C who put Sri Lanka on the world map of paediatrics. Soon after completing his autobiography he passed away peacefully on 20th of May 1987.

For the Prof. C.C. de Silva memorial oration this year, I have chosen the topic 'The child with urinary tract infection: a dilemma for the paediatrician'.

\section{Introduction}

Urinary tract infections (UTIs) are some of the most common and serious infections of childhood, particularly in the early years of life. Apart from

${ }^{1}$ Senior Lecturer and Head of Department, Faculty of Medicine, University of Peradeniya.

causing a potentially serious acute illness, UTIs can damage the kidneys and cause renal scarring, leading to hypertension and reduced renal function in later life $\mathrm{e}^{1,2}$. Interestingly, UTIs in the pre-antibiotic era were primarily treated by disinfecting the colon. The scientific rationale of this therapeutic approach was the suspicion that bowel flora was responsible for causing UTIs. This is true even in the 21 st century. Sterilization of the bowel was performed by using large enemata and calomel. Moreover, routine appendicectomy was performed if there was any suspicion of involvement of the appendix. If cystitis was suspected, washing out of the bladder was performed. In cases of pyelonephritis, urine was alkalinized by using large doses of citrate of potash. In more chronic cases they recommended use of a vaccine prepared from the urine of the patient. In resistant cases the last option was to improve the child's general health with a change in environment ${ }^{3}$. The acute stage morbidity and mortality of patients with UTIs were significantly high during this period. In 1931, John Thompson reported 3 deaths out of 24 cases of pyelitis in infancy. Jeffreys reported 6 deaths out of 60 cases, in all ages, treated at Great Ormond Street Children's Hospital, London.

With the discovery of antibiotics in the early 1940's, there was a drastic reduction in the acute morbidity and mortality of childhood UTIs. Presently, the major concerns of the medical fraternity are focused on the long term effects of $\mathrm{UTI}^{4}$. Recent technological advances in imaging techniques, such as ultrasonography, x-rays and radioisotope scanning, have highlighted the long -term effects of $\mathrm{UTI}^{5}$. There are many case reports that highlight new scar formation following UTIs detected using both intravenous urography and DMSA scans ${ }^{6,7}$. Several independent groups have confirmed link between UTI, vesicoureteric reflux (VUR) and renal scarring ${ }^{4,8}$. Smellie et al have also demonstrated a link between delay in diagnosis and treatment and the development of new renal scars. The risk of developing UTI before the age of 14 years is approximately $1 \%$ in boys and $3-5 \%$ in girls ${ }^{2}$. Correct diagnosis and management of UTIs is of utmost 
importance in order to prevent potential renal parenchymal damage that can lead to chronic renal failure in later life $\mathrm{e}^{4,9}$.

VUR is a risk factor contributing to the development of UTIs and is present in a third of young children with $\mathrm{UTI}^{10,11}$. It is also a risk factor for renal scarring, with the propensity to progress towards reflux nephropathy ${ }^{12,13}$. It is also associated with renal dysplasia and other developmental abnormalities of the urinary tract including detrusor instability. Evidence suggests that an autosomal dominant mechanism is responsible for causing reflux and renal scarring in certain children ${ }^{14}$. With increase in knowledge, a consensus statement was produced in 1991 by the Royal College of Physicians to detect and manage UTIs in children ${ }^{15}$. Paediatricians in many countries have followed these guidelines. I became particularly interested in the management of childhood UTI in the early 1990's.

Although UTI is a common problem, lack of awareness of the presenting features, limited access to laboratory facilities and indiscriminate use of antimicrobial therapy may have resulted in continuing infections leading to scarring of kidneys. These effects are witnessed by adult nephrologists who see many progressing to end stage renal failure today. Establishing new nephrology units to serve increasing numbers of adult patients seeking treatment for renal failure, long waiting lists for costly procedure of renal transplantation plus frequent paper advertisements appealing for financial assistance may reflect our poor practice in the past. This evening I wish to present to you the findings of a prospective study which was undertaken at the Paediatric Unit, Peradeniya, from 1993 onwards in order to evaluate the impact of UTIs in the acute stage and its long-term effects.

The objective of the study was to establish data for UTIs in regard to presenting features, usefulness of urine microscopy in the diagnosis, antibiotic sensitivity pattern of the causative organisms, pattern of underlying urinary tract anomalies, predisposing factors for renal scarring and long-term outcome.

\section{Patients and Method}

Patients admitted to the paediatric ward and patients referred to the renal clinic with bacteriologically confirmed UTIs were studied. Their details were entered in a computer database and followed up in a special weekly clinic under personal supervision. Parents and guardians of children were educated about the disease and its long-term management. An educational booklet was provided free.

Infection of the urinary tract was identified by the growth of a significant number of organisms of a single species in a properly collected sample of urine, in the presence of symptoms. It was difficult to differentiate clinically between upper and lower UTIs. Thus, all febrile infections were considered to involve the upper urinary tract with potential for scarring. It was necessary to have a high degree of suspicion since their presenting features were nonspecific. All infants and children presenting with unexplained fever and neonates with septicaemia were suspected to have a UTI. Infants and young children who presented with recurrent fever, prolonged diarrhoea, vomiting, abdominal pain, poor weight gain and children having urinary symptoms were investigated to exclude UTI. Confirmation of the diagnosis was made on positive urine cultures in properly collected samples of urine. Almost always attempts were made to collect 2 samples of urine before instituting antimicrobial therapy. Moreover, clear instructions were provided to parents and nursing staff regarding method of collection and transport of specimens in order to minimise the chances of contamination. Urinalysis was helpful in providing immediate information and helped initiation of treatment. Clean catch midstream specimens of urine were collected in most cases after washing the genitalia with soap and water. Specimens were collected into sterile, screw capped glass bottles. In neonates and infants, urine samples were obtained by suprapubic aspiration or by clean catch specimens. Collection of specimens into a sterile bag strapped to the perineum was discouraged as risk of contamination was high. Urine cultures were repeated if contamination was suspected and if colony counts were equivocal. Definitions are given in Table 1. 
Tabie 1

Definitions $^{16}$

\begin{tabular}{|l|l|}
\hline Significant Bacteriuria & Colony count of $>105 / \mathrm{ml}$ of a single species in a midstream clean catch sample \\
\hline Asymptomatic bacteriuria & $\begin{array}{l}\text { Presence of significant bacteriuria in two or more specimens of a child with no } \\
\text { symptoms }\end{array}$ \\
\hline Recurrent UTI & Second attack of UTI \\
\hline Complicated UTI & $\begin{array}{l}\text { Presence of fever }>38.50 C, \text { toxicity, persistent vomiting, dehydration and renal } \\
\text { angle tenderness. }\end{array}$ \\
\hline Simple UTI & $\begin{array}{l}\text { UTI with low-grade fever, dysuria, frequency, urgency but none of the above } \\
\text { symptoms }\end{array}$ \\
\hline
\end{tabular}

A careful evaluation of the child was done to detect the severity, complications and risk of recurrence. Inquiry was made regarding the bowel and urinary habits that indicate an obstruction to urinary outflow. The abdomen was palpated for renal masses and the genitalia examined for evidence of infection, phimosis, tight prepuce or fused labia. Blood pressure was recorded. A detailed neurological examination was performed if there were symptoms of a neurogenic bladder. Full blood count, blood culture, serum electrolytes and estimation of urea and creatinine were done in children with complicated infections. If the response to treatment was slower than anticipated or if clinical evidence of urinary tract obstruction existed an urgent ultrasound scan was performed.

\section{Treatment}

The patient's age, general condition and likelihood of compliance with medication helped in deciding between outpatient therapy and hospitalization. Antibiotics were given parenterally to infants and children with complicated infections (Table 2). Treatment was initiated using a second or a third generation cephalosporin or co-amoxyclav until culture reports were available. Change over to an oral antibiotic was done if the child showed clinical improvement. Babies under 3 months and children with a positive blood culture were treated with parenteral antibiotics for the entire duration. Children above 3 months with a simple infection were treated with oral antibiotics (Table 2). Cephalosporins and co-amoxyclav that reach the renal parenchyma and blood stream in adequate therapeutic concentrations were selected. Nitrofurantoin and nalidixic acid were not considered since they did not reach adequate tissue levels. Repeat urine culture and ultrasound scan of the urinary tract were performed in children who did not show an improvement within 48 hours. Treatment was given for 10-14 days for children with complicated infections and 7-10 days for simple infections. All the children were investigated to identify underlying anomalies leading to recurrent infections and renal damage. Guidelines for evaluation of patients varied with age (Figure 1).

Table 2

Antimicrobials for treatment of UTI

\begin{tabular}{|l|l|l|l|l|l|}
\hline & Parenteral & & & Oral & \\
\hline Medication & $\mathrm{mg} / \mathrm{kg} / \mathrm{day}$ & Doses/day & Medication & $\mathrm{mg} / \mathrm{kg} /$ day & Doses/day \\
\hline Cefuroxime & $75-100$ & 3 & Amoxicillin & $30-35$ & 3 \\
\hline Gentamicin & $5-6$ & 2 & Cotrimoxazole & $\begin{array}{l}6-10 \\
\text { (trimethoprim) }\end{array}$ & 2 \\
\hline Co-amoxiclav & $\begin{array}{l}75-100 \\
\text { (amoxicillin) }\end{array}$ & $2-3$ & Cephalexin & $50-70$ & 3 \\
\hline Cefotaxime & $100-150$ & 3 & Co-amoxiclav & $\begin{array}{l}75-100 \\
\text { (amoxicillin) }\end{array}$ & $2-3$ \\
\hline Ceftriaxone & $75-100$ & $1-2$ & Cefaclor & 40 & 3 \\
\hline
\end{tabular}




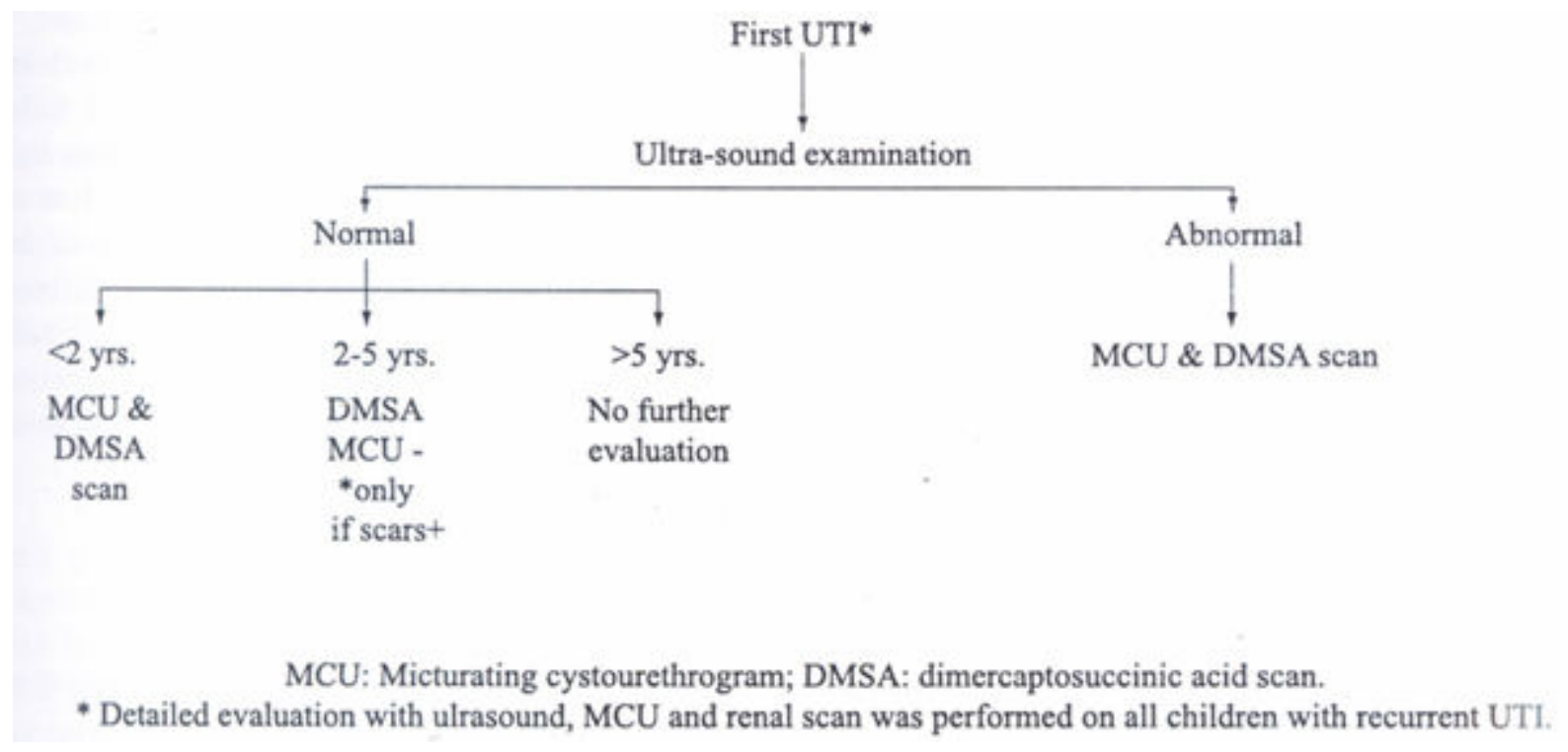

Figure 1 Evaluation following initial UTI.

DTPA scans were performed in children with evidence of hydronephrosis in the absence of reflux. This was useful in assessing the function of kidneys and drainage of the collecting system. In selected cases, X-rays of the kidneys and the bladder area were taken to exclude radio-opaque calculi. Cystoscopy and intravenous pyelograms were rarely performed. Urine cultures were routinely repeated at cessation of antibiotic therapy. A detailed analysis was made of all the urine culture reports between two yearly periods for a comparative analysis. Prophylactic antibiotics were given to all children with UTIs until the imaging studies were completed. If there was a reflux or renal scarring prophylaxis was continued until five years. Children with recurrent infections were also given prophylactic antibiotics for a period of two years even if the urinary tract was normal. Nitrofurantoin, nalidixic acid, cephalexin and cotrimoxazole were used as prophylactic drugs and given as a single bedtime dose (Table 3).

Table 3

Antimicrobials for prophylaxis

\begin{tabular}{|l|l|}
\hline Drug & $\mathrm{mg} / \mathrm{kg} /$ day \\
\hline Nitrofurantoin & $1-2$ \\
\hline Nalidixic acid & 12.5 \\
\hline Cephalexin & 10 \\
\hline Cotrimoxazole & $1-2$ (trimethoprim) \\
\hline
\end{tabular}

Plenty of fluids were encouraged. Frequent voiding and the practice of double micturition was recommended to minimise stasis of urine in the bladder. High fibre diet was encouraged. Attention to undergarments and perineal hygiene, including foreskin of boys, was explained to parents. Although circumcision was not routinely done it was considered in children having a reflux with recurrent infections. Asymptomatic bacteriuria is a benign condition. Organisms commonly identified are usually low virulent E. coli. In many instances eradication of low virulent bacteria is followed by symptomatic infection with more virulent bacterial strains. Hence antibiotic therapy was not prescribed.

Among children with UTIs, VUR is seen in about 30$50 \%$, while in infants it is $40-50 \%$. Presence of moderate to severe reflux is known to predispose towards reflux nephropathy. International reflux study classification helps to grade reflux from I-V on severity ${ }^{17}$. Basis for classification is the appearance of the urinary tract in micturating cystourethrogram (MCUG). Children with high grade reflux and concomitant infections are at a higher risk for renal scarring. Reflux generally resolves or improves with bladder growth and maturation ${ }^{18,19}$. Lesser grades of reflux are more likely to resolve than higher grades. A majority of children were managed on long term antibiotics with the expectation of spontaneous resolution of reflux. All patients were closely monitored for breakthrough infections. The prophylactic antibiotics were discontinued in children above five years despite the presence of mild to moderate reflux. According to published data risk of recurrent infections and renal scarring are low after four to five years ${ }^{20}$. Surgical repair was considered in 
patients with Grade V reflux or if breakthrough infections developed in patients with Grade IV reflux. After surgery, prophylactic antibiotics were continued for six months. Treatment of primary VUR is outlined in Table 4.

Table 4

Treatment of primary vesicoureteric reflux

\begin{tabular}{|l|l|l|}
\hline Grade of reflux & Medical treatment & Surgical treatment \\
\hline Grade I, II, III & Antibiotic prophylaxis up to 5 years of age & \\
\hline Grade IV & Antibiotic prophylaxis up to 5 years of age & Surgery if breakthrough infections occur \\
\hline Grade V & Antibiotic prophylaxis until surgery & In all patients \\
\hline
\end{tabular}

All children were followed up under personal supervision. Patients were monitored for their physical growth, renal function and blood pressure. Urine cultures were done promptly if they were symptomatic. Children with renal scars were given continuous care up to adulthood. Children without underlying abnormalities were given the opportunity to attend the clinic if they became symptomatic. All patients with reflux who have completed 5 years of follow up were reviewed and assessed for long-term outcome.
Over a period of ten years from 1993 onwards 808 children with confirmed UTIs were registered in our clinic. $447(55 \%)$ were males and $361(45 \%)$ were females. Male: female ratio was 1:2. $672(83 \%)$ of them were below the age of 5 years. There were more males than females below one year of age. Sex ratio was almost equal in older children (Figure 2). Fever was the main presenting feature in $81 \%$. It is interesting to note that only around one third of children presented with symptoms relating to the urinary tract (Table 5).

\section{Results $^{21}$}

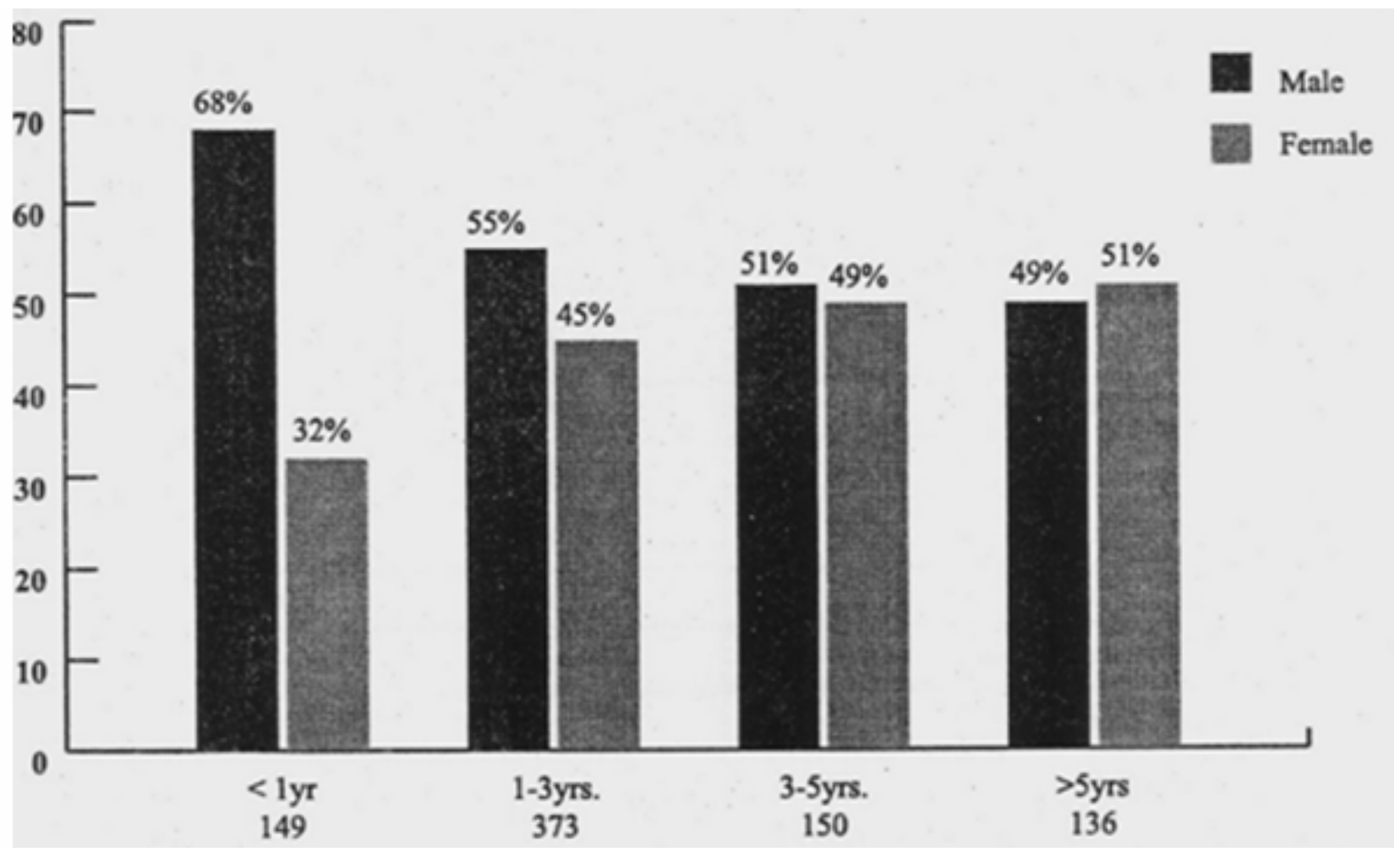

Figure 2 Age and sex distribution 
Table 5

Presenting features

\begin{tabular}{|l|c|}
\hline \multicolumn{1}{|c|}{ Presenting complaint } & Percentage \\
\hline Fever & $81 \%$ \\
\hline Dysuria & $30 \%$ \\
\hline Vomiting & $29 \%$ \\
\hline Frequency of micturition & $25 \%$ \\
\hline Excessive crying & $24 \%$ \\
\hline Diarrhoea & $12 \%$ \\
\hline Neonatal jaundice & $04 \%$ \\
\hline Febrile convulsions & $03 \%$ \\
\hline
\end{tabular}

2062 urine culture and antibiotic sensitivity reports were analyzed for from January to December1997 of which $8.3 \%$ had significant colony counts. From November 2001 to October 2002 (one year), a total of 2650 culture reports were analyzed of which $10.4 \%$ had significant colony counts. In both groups $65 \%$ were males.

In 2002 co-amoxiclav had the highest antibiotic sensitivity of $87 \%$. Nitrofurantoin, nalidixic acid and norfloxacin had high sensitivities both in 1997 and 2002 which were statistically significant when compared to ciprofloxacin and mecillinam which had low sensitivity levels (Table 6). A change was evident in sensitivity levels of cotrimoxazole and cephalexin. These had low sensitivities in 1997 and relatively higher sensitivities in 2002. In 1997 sensitivity tests were not performed for co-amoxyclav and cefradine.

Table 6

Antibiotic sensitivity patterns

\begin{tabular}{|l|c|c|}
\hline \multicolumn{1}{|c|}{ Antibiotic } & $\begin{array}{c}1997 \% \\
\text { Sensitivity }\end{array}$ & $\begin{array}{c}2002 \% \\
\text { Sensitivity }\end{array}$ \\
\hline Nalidixic acid & 73.18 & 76.59 \\
\hline Nitrofurantoin & 73.15 & 80.03 \\
\hline Cotrimoxazole & 40.16 & 63.83 \\
\hline Norfloxacin & 66.95 & 75.68 \\
\hline Cephalexin & 54.84 & 69.16 \\
\hline Ciprofloxacin & 51.85 & 47.37 \\
\hline Mecillinam & 35.48 & 33.78 \\
\hline Ampicillin & 58.33 & 58.06 \\
\hline Gentamicin & 50.00 & 60.71 \\
\hline Co-amoxiclav & - & 86.67 \\
\hline Cefaclor & - & 52.72 \\
\hline
\end{tabular}

Ultrasound scans were performed in all 808 children, of which $26 \%$ showed abnormalities. $41.6 \%$ of patients were subjected to MCUG of which $30 \%$ were abnormal. $48 \%$ of them had undergone DMSA scans of which $24 \%$ showed scarring of kidneys. Abnormalities were seen in $24.6 \%$ of DTPA scans performed in $16 \%$ (Table 7 ).

Table 7

Results of the imaging studies

\begin{tabular}{|l|c|c|}
\hline Investigation & Number & $\begin{array}{c}\text { Number with } \\
\text { anomalies }\end{array}$ \\
\hline MCUG & $335(41.46 \%)$ & $101(30 \%)$ \\
\hline DMSA & $388(48.02 \%)$ & $93(24 \%)$ \\
\hline DTPA & $130(16.09 \%)$ & $32(24.6 \%)$ \\
\hline USS & $808(100 \%)$ & $213(26 \%)$ \\
\hline
\end{tabular}

The commonest abnormality detected was renal scarring (Table 8). 23\% had demonstrable renal scars and of them $61 \%$ were below one year. Interestingly, 44 patients had renal scarring without evidence of reflux. Reflux was demonstrated in 19\%. A majority had minor grades of reflux (I - III). $9 \%$ and $3 \%$ of patients showed evidence of Grade IV and V reflux. Of 155 patients with demonstrable reflux in MCUG, ultrasound scan detected reflux in 85 patients establishing a sensitivity of $55 \%$. In 44 patients ultrasound scan revealed VUR, but MCUG was normal with a specificity of $72 \% .6 .6 \%$ showed evidence of partial pelvi-ureteric junction obstruction on DTPA scan but IVP showed a definite obstruction in only $1.5 \%$. Posterior urethral valves were detected in $2 \% .88 \%$ of them were below the age of one year, which constituted $18 \%$ of the total study. It is unfortunate that 16 patients with posterior urethral valves presenting with UTIs were detected in later life but most of these patients would have been diagnosed ante-natally in developed countries. Other minor abnormalities detected were duplex system, absent kidneys, horseshoe kidneys, neurogenic bladder, dysplastic and pelvic kidneys. 
Table 8

Underlying abnormalities ${ }^{22}$

\begin{tabular}{|l|c|c|}
\hline \multicolumn{1}{|c|}{ Abnormalities } & Total number & Age below one year \\
\hline VUR & $155(19.18 \%)$ & $95(61.2 \%)$ \\
\hline Renal scars & $183(22.6 \%)(\mathrm{VUR}+$ in 139$)$ & $113(61.7 \%)$ \\
\hline Pelvi-ureteric junction obstruction & $49(6.6 \%)$ & $9(18 \%)$ \\
\hline Posterior urethral valves (PUV) & $16(1.98 \%)$ & $3(87.5 \%)$ \\
\hline Hypoplastic kidneys & 5 & 0 \\
\hline Pelvic kidneys & 2 & 0 \\
\hline Horse shoe kidneys & 1 & 1 \\
\hline Absent kidneys & 1 & 0 \\
\hline Ureteric diverticulum & 1 & 0 \\
\hline Bladder diverticulum & 1 & 0 \\
\hline Congenital megaureter & 1 & 1 \\
\hline Bifid pevicalyceal system & 2 & \\
\hline
\end{tabular}

\section{Analysis of children with renal scars ${ }^{23}$}

$22.6 \%$ of children in the study group had detectable renal scars on DMSA scans. $52 \%$ of them were males and $48 \%$ were females. $76 \%$ had scarring of upper poles of kidneys. $24 \%$ of them had multiple scars with 7\% having bilateral involvement (Table 9). Over $70 \%$ were below 5 years and $42 \%$ were below 2 years (Figure 3). 92\% of children were detected during their first presentation. Only $66 \%$ presented with specific symptoms. $45 \%$ of children with renal scars had underlying abnormalities, and the main abnormality detected was reflux in $72 \%$ (Table 10 ).
E.coli was the commonest organism cultured from $95 \%$.

Table 9

Degree of renal scarring

\begin{tabular}{|c|c|}
\hline Degree of scarring & Percentage \\
\hline Right upper pole scarring & $32 \%$ \\
\hline Left upper pole scarring & $44 \%$ \\
\hline Multiple scars & $24 \%$ \\
Right kidney & $6 \%$ \\
Left kidney & $11 \%$ \\
Bilateral & $7 \%$ \\
\hline
\end{tabular}

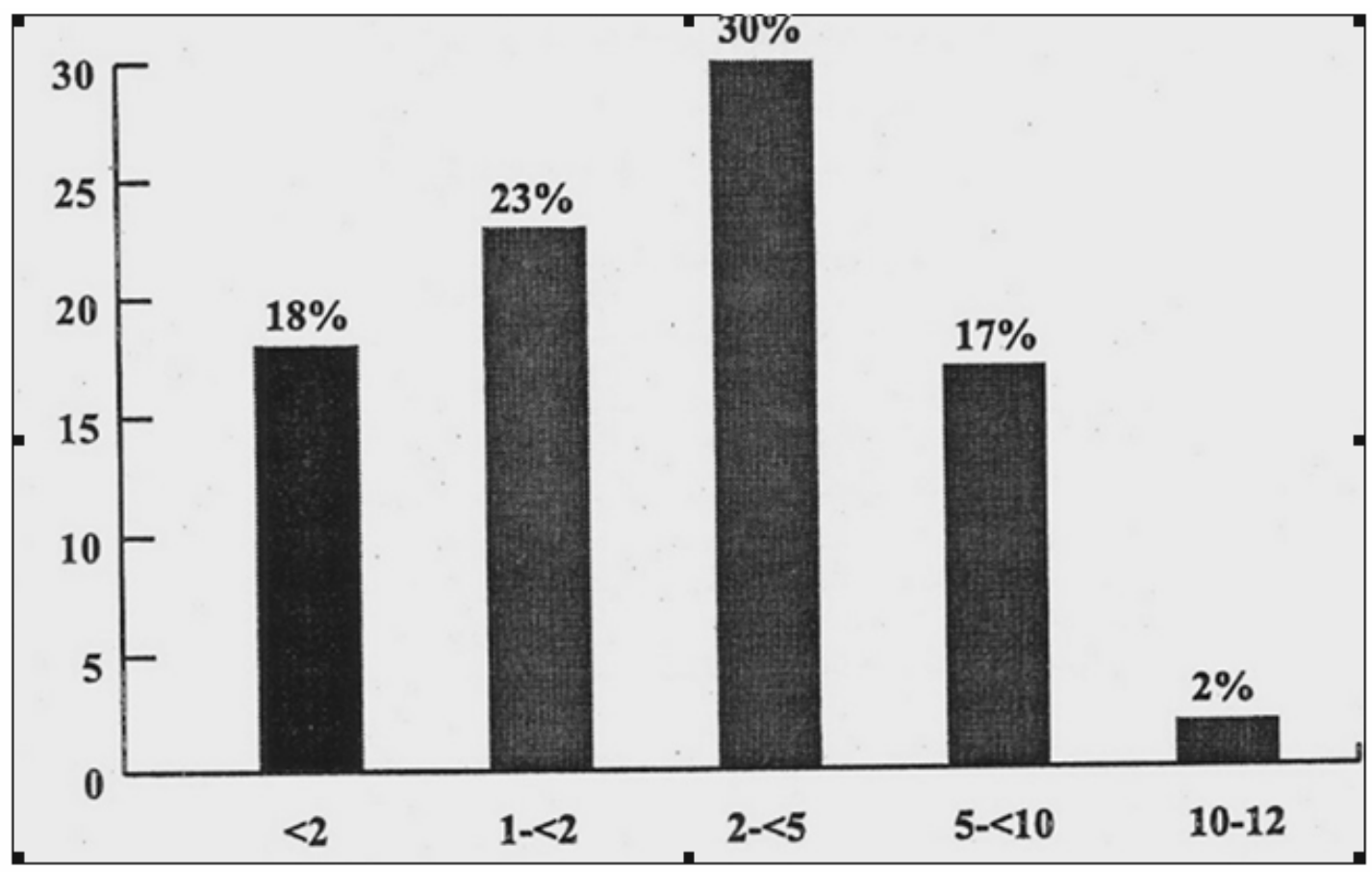

Figure 3. Age distribution of children with scars 
Table 10

Underlying abnormalities seen in children with scarring

\begin{tabular}{|l|c|}
\hline \multicolumn{1}{|c|}{ Abnormality } & Percentage \\
\hline VUR & 72 \\
\hline PUJ obstruction & 9 \\
\hline PU valves & 9 \\
\hline Neurogenic bladder & 4 \\
\hline Horse-shoe kidney & 4 \\
\hline Duplex system & 2 \\
\hline
\end{tabular}

Long term follow up study of children with vesicoureteric reflux ${ }^{24}$

Fifty nine patients with initial reflux confirmed by MCUG were studied. They were followed up for 612 years with the average being 8 years. Male: female ratio was $1.6: 1$ and mean age at presentation was 1.9 years. Their long- term outcome was assessed in regard to occurrence of UTIs during and after discontinuation of prophylaxis, adverse effects to prophylactic drugs, clinical and laboratory evidence of renal failure, such as blood pressure measurements, physical growth parameters and proteinuria. A majority had Grade III VUR (Figure 4). Reflux was secondary to posterior urethral valves in $3 \% .52 \%$ of children with reflux had detectable renal scars. $15 \%$ of this group underwent surgery, of which over $75 \%$ were due to anomalies other than reflux such as phimosis, posterior urethral valves and hypospadias. Blood pressure measurements were taken at the end of the follow up period (Table 11).

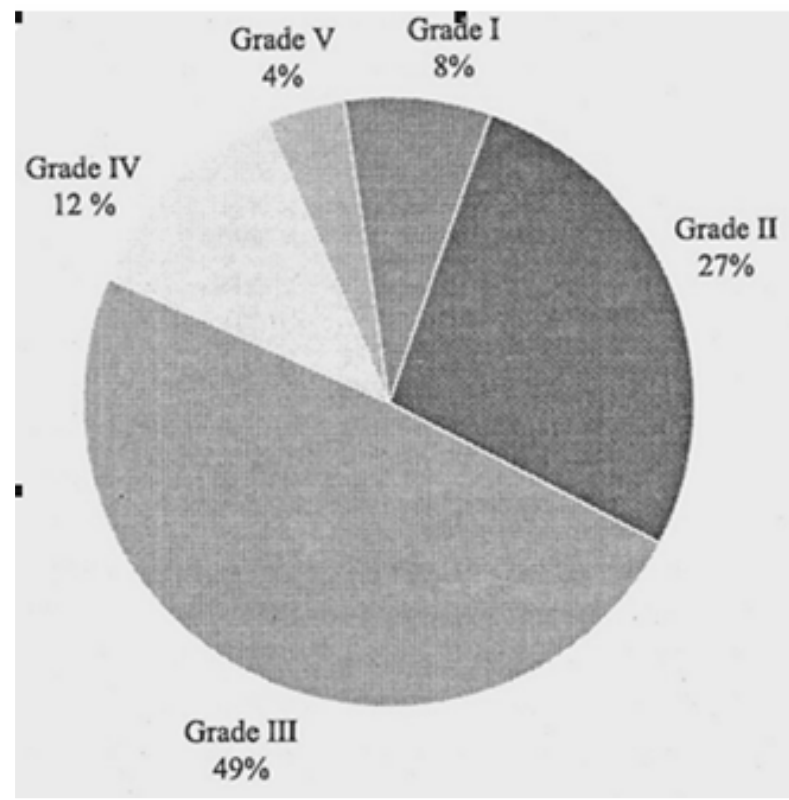

Figure 4 Grading of VUR
Table 11

Measurements in patients with VUR at the end of follow up ${ }^{25}$

\begin{tabular}{|l|c|c|}
\hline \multicolumn{1}{|c|}{$\begin{array}{c}\text { Age-specific } \\
\text { percentile of BP }\end{array}$} & SBP & DBP \\
\hline Below 5th & $16.6 \%$ & $3.7 \%$ \\
\hline 5 th-10th & $16.6 \%$ & 0 \\
\hline 11th-25th & $5.6 \%$ & $3.7 \%$ \\
\hline 26-50th & $31.5 \%$ & $16.6 \%$ \\
\hline 51 st -75 th & $22.2 \%$ & $29.6 \%$ \\
\hline 76 th -90 th & $5.6 \%$ & $37.0 \%$ \\
\hline 91st -95 th & $1.9 \%$ & $1.9 \%$ \\
\hline
\end{tabular}

One patient was on long-term antihypertensives. All other patients recorded systolic blood pressures below 95th percentile for age. Urine was free of proteins in a majority. 3 patients had laboratory evidence of established renal failure. Two of them had presented before the age of two years with posterior urethral valves and are awaiting renal transplant. The other patient whose renal failure was due to a neurogenic bladder underwent a renal transplant at the Teaching Hospital, Peradeniya, with the help of Dr. Oswald Fernando from the UK. This is the first paediatric renal transplant done in our country.

\section{Discussion}

In this study 808 children with culture positive UTIs were followed up over a ten year period. Special emphasis was given to the accuracy of initial diagnosis. In spite of stringent entry criteria, the large number of cases studied illustrates how common UTIs are in paediatric practice. More than $75 \%$ in this study were below five years. Similar observations have been made locally by Lamabadusuriya $^{26}$, de Silva et $\mathrm{al}^{27}$ and David ${ }^{28}$ who showed that more than $55 \%$ of children in their studies were below five years. This indicates that young children are more at risk. This study group shows a male preponderance below one year, which is in keeping with the figures from the West. Lamabadusuriya in his study of 165 patients followed up in private sector hospitals and de Silva et al in their study made similar observations.

Fever was the commonest presenting feature in $70 \%$. Only $30 \%$ presented with symptoms relating to the urinary tract. Observations made by Lamabadusuriya, de Silva and David in their studies are similar. Therefore, in children presenting with fever without associated symptoms, a high degree of suspicion is necessary for diagnosis. $27 \%$ did not show any significant microscopic abnormalities indicating that 
the "gold standard" for establishment of diagnosis is a positive urine culture. E. coli was the commonest organism isolated from $95 \%$ in our study.

Co-amoxyclav, which had the highest level of sensitivity, along with cephalosporins, remain the most suitable tissue penetrating antibiotics for treatment of acute episodes. Nalidixic acid and nitrofurantoin have retained their high levels of sensitivities. These antibiotics therefore remain suitable for the treatment of lower UTIs and for prophylaxis. However, ciprofloxacin and mecillinam had low levels of sensitivities indicating that resistance had built up against these two drugs. Therefore prescribing of these two drugs is not recommended. Gentamicin, which had a low level of sensitivity in 1997, had attained a somewhat acceptable sensitivity level in 2002. Use of gentamicin for the treatment of acute episodes has diminished over the last few years due to nephrotoxicity and availability of safer alternatives such as cephalosporins. This decrease in usage is the most likely cause for its increased sensitivity in 2002. The change in sensitivity pattern of cotrimoxazole over the 5-year period was noteworthy. This emphasizes the importance of periodic revision of antibiotic sensitivity patterns to enable appropriate treatment.

The study shows that ultrasound scan was not sensitive in detecting VUR. Gunaratna et $\mathrm{al}^{29}$ in their study have made similar observations. VUR was the commonest abnormality detected in $20 \%$. Similar observations have been made by Gunaratna et.al in their study where they have performed 658 MCUGs in children with UTIs. Lamabadusuriya, de Silva et al and David have made similar observations. This reflects the trend in the West.

$23 \%$ of children had detectable renal scarring. Smellie et $\mathrm{al}^{30}$ observed renal scarring in $5-20 \%$ of children after UTI. Their numbers are based on findings seen in intravenous urograms. It is a less sensitive method of detecting renal scars than DMSA scans. Recent studies done in Sweden by Jacobson et $\mathrm{al}^{31}$ using DMSA scans show an incidence of $37 \%$. $70 \%$ of children with renal scars were below five years. This finding is in keeping with the studies of Smellie et. al which showed that infants and children less than five years are at greater risk of developing new scars. $90 \%$ with renal scars presented with the first episode. A thorough evaluation of the histories of these patients showed that some of them have had recurrent febrile episodes in early infancy. It is possible that they may have had undetected infections during infancy, masked by indiscriminate use of antibiotics. It is important to note that $35 \%$ presented with nonspecific symptoms. A high degree of suspicion is necessary to identify patients at risk of renal damage.

The main abnormality detected was VUR in $75 \%$ of patients with scars. Although reflux or other structural abnormalities have been considered as prerequisites for the development of renal scars, only $33 \%$ of the children had demonstrable reflux. This study confirms the observations made by Jacobson et al that renal scarring occurs very often without demonstrable reflux or obstruction. There may be other unidentified host or bacterial factors responsible. In some of these patients reflux may have resolved before presentation. The other explanation is that reflux can be intermittent. Results of this study highlight the importance of imaging in children with confirmed UTIs, especially in the group below one year.

Unlike in developed countries where many urinary tract anomalies are detected with antenatal scanning, most of our patients present with symptomatic infections, particularly those with posterior urethral valves. Moreover, as a result of indiscriminate use of antibiotics for febrile illnesses in childhood, coupled with the lack of culture facilities in primary health care centres, renal scarring may be undiagnosed in children with UTI.

Over the years evidence confirms that renal scarring is an unlikely complication of UTIs in patients over 5 years. Hence the international consensus is to carry out minimal imaging for this group ${ }^{20,32}$. However, in the Sri Lankan context with limited diagnostic facilities, it may be prudent to carry out a DMSA scan to detect renal scarring even in patients presenting above 5 years. The presence of renal scars can predispose the child to hypertension and chronic renal failure ${ }^{31}$. Our patients with reflux were managed conservatively with regular monitoring and administration of prophylactic antibiotics except in 6 patients. Complications such as hypertension and progression to end stage renal failure were rare. These findings reflect recent reports of excellent outcome in children with reflux managed conservatively.

Due to concern of reflux nephropathy, the Royal College of Physicians published guidelines in 1991 recommending imaging in every child after the first $\mathrm{UTI}^{15}$. The working group advised all patients to have an ultrasound scan and that the DMSA scan should be carried out on those below 7 years. MCUG was considered mandatory in children below 1 year. 
Without good evidence of benefits from treatment plus poor compliance to long-term low dose prophylaxis, the presumed benefits of imaging and long-term treatment may not be as good as originally expected. Stark ${ }^{33}$, in 1997, challenged the validity of imaging tests and said they are excessively costly and invasive without any benefit.

Tertiary specialists were heavily represented in the research-working group of the Royal College of Physicians. Their views and experiences represented the consensus on UTIs and reflux nephropathy. In contrast, general practitioners and paediatricians, who see most children during their first UTI, were in the minority. However, published guidelines provided a useful step in evaluating current practice. These guidelines are followed extensively in UK and Sri Lanka. However, ironically and most unfortunately, studies suggest that many children do not have the recommended management.

Although there was no formal attempt to inform general practitioners of the guidelines, some general practitioners are now referring large numbers of children for imaging investigations and a paediatric opinion following simple, non-febrile UTI. This has generated a massive workload for radiology departments and exposed large numbers of children to significant radiation without much evidence of benefit. The proportion of children seen with evidence of renal damage is lower than in earlier studies. This may be because UTI is diagnosed early in infants when they are referred to hospital, or it may reflect the fact that many more straightforward cases are being referred for further investigation. Unfortunately, there is continuing evidence of delay in diagnosis of UTI in infants and toddlers in primary care where urine collection is perceived as difficult and some children have several consultations before the diagnosis of UTI is considered and further delay before it is confirmed.

In conclusion, it is now clear that evidence based recommendations, even for the developed countries have yet to be reached. The situation is worse for developing countries where the facilities are not readily available. This poses a therapeutic dilemma for the practising paediatrician. In Sri Lanka certain towns have collection centres for urine cultures. These are sent to established laboratories in Colombo. Accuracy of results is likely to suffer adversely during transport, as the diagnosis of UTI is heavily dependant on the results of the urine culture report. Thus the paediatrician is left in a dilemma with regard to the extent of imaging of the urinary tract. On one hand imaging is costly, invasive and involves irradiation of a vital area of the child which can be totally unnecessary if the urine culture is falsely positive. On the other hand lesser investigations may miss significant underlying anomalies. It is worth emphasising here that infants and young children with neglected UTIs may end up with renal failure. Hence it is a worthy asset to improve the microbiological infrastructure facilities in the lesser developed districts of Sri Lanka which would facilitate the proper diagnosis of children with UTIs, particularly the very young patients. Until such time children with suspected UTIs could be referred to centres where they could be properly investigated and managed, thus reducing the potential risk of end stage renal failure.

\section{References}

1. Roberts K B, Charney E, Sweren R J. Urinary tract infection in infants with unexplained fever: a collaborative study. J Pediatr. 1983; 103:8647.

2. Winberg J, Andersen H J, Bergstrom T, et al. Epidemiology of symptomatic urinary tract infection in childhood. Acta Paediatr Scand. 1974; 252:1-20.

3. Hutchison R. editor. Lectures on Diseases of children. 6th ed. Great Ormond Street London, Edward Arnold \& Co.; 1931:375-86.

4. Smellie J M, Poulton A, Prescod N P Retrospective study of children with renal scarring associated with reflux and urinary infection. Br Med J 1994; 308:1193-6.

5. Vernon S J, Coulthard M G, Lambert H J, Keir M J, Matthews J N S. New renal scarring in children who at age 3 and 4 years had had normal scans with dimercaptosuccinic acid: follow up study. BMJ 1997; 315:905-8.

6. Smellie J M, Ransley P G, Normand I C S, Prescod N, Edwards D. Development of new renal scars; a collaborative study. $B M J 1985$; 290: $1957-60$.

7. Winberg J, Bollgren I, Kallenius G, Mollby R, Svenson S B. Clinical pyelonephritis and focal renal scarring. A selected review of pathogenesis, prevention and prognosis. Paediatr Clin North Am 1982; 29:801-4. 
8. Becker G J, Kincaid-Smith P. Reflux nephropathy: the glomerular lesion and progression of renal failure. Pediatr Nephrol 1993; 7:365-9.

9. Winter A L, Hardy B E, Alton D J, Arbus G S, Churchill B M Acquired renal scars in children. $J$ Urol. 1983; 129:1190-4.

10. Hellerstein S Recurrent urinary tract infections in children. Pediatr Infect Dis. 1982; 1:271-81.

11. Hashemi G Recurrent urinary tract infection. Indian J Pediatr. 1985; 52:401-3.

12. Anon. Vesico-ureteric reflux [editorial]. Lancet 1968; i: 1072-4.

13. Becker G J, Kincaid-Smith P. Reflux nephropathy: the glomerular lesion and progression of renal failure. Pediatr Nephrol 1993; 7:365-9.

14. Kenda R B, Fettich J J. Vesicoureteric reflux and renal scars in asymptomatic siblings of children with reflux. Arch Dis Child 1992; 67:506-8.

15. Report of a working group of the research unit; Royal College of Physicians. Guidelines for the management of acute urinary tract infection in childhood. Journal of the Royal College of Physicians of London 1991; 25: 36-42.

16. North A F. Bacteriuria in children with acute febrile illnesses. J Pediatr. 1963; 63:408-11.

17. International Reflux Study in Children. International system of radiographic grading of vesicoureteric reflux. Paediatric Radiology 1985; 15:105-9.

18. Edwards D, Normand I C S, Prescod N, Smellie J. Disappearance of vesicoureteric reflux during long-term prophylaxis of urinary tract infection in children. $B M J 1977$; ii: 285-8.

19. Smellie J M. The disappearance of reflux in children with urinary tract infection during prophylactic chemotherapy. In: Alwall N, Berglund F, Josephson B, editors. Proceedings of the 4th International Congress of Nephrology, Stockholm 1969, Vol 3. Clinical nephrology, immunology. Basel: Karger, 1969; 357.
20. American Academy of Pediatrics. Practice parameter: The diagnosis, treatment and evaluation of the initial urinary tract infection in febrile infants and young children. Pediatrics 1999; 103: 843-52.

21. Abeysekera C K, Jayasena L, Abeyagunawardena A S. A study of urinary tract infections in childhood. Proceedings of the Annual Congress of Kandy Society of Medicine. February 1995; Kandy, Sri Lanka.

22. Abeysekera C K, Jayasena L. An analysis of abnormalities in 325 children with urinary tract infections. Proceedings of the second Annual Scientific Congress of Sri Lanka College of Paediatricians. 1998 July 15-19; Colombo, Sri Lanka.

23. Abeysekera C K. Renal scarring in children with urinary tract infections. Sri Lanka Journal of Child Health, 2000; 29: 85-7.

24. Yasaratne B M G D, Abeysingha C K, Manuwickrama $\mathrm{T}$ D, Abeysekara C $\mathrm{K}$, Abeyagunawardena A S. Long term follow up study of children with vesicoureteric reflux. Proceedings of Annual Scientific Congress of Sri Lanka College of Paediatricians 2004 Aug 1114; Kandy, Sri Lanka.

25. Smith G C, Inward C. How and when to measure blood pressure. In: Webb N J A, Postlethwaite R $\mathrm{J}$, editors. Clinical paediatric nephrology. $3 \mathrm{rd}$ ed. Oxford, 2003:35-150.

26. Sanath Lamabadusuriya. A prospective study of urinary tract infections in children. Sri Lanka Journal of Child Health 2001; 30(2); 31-7.

27. Silva D D S, Dissanayake D M R, Sathkumara S M S P, Jayawardena S D, Athukorala S D. Patterns of abnormalities detected in children with urinary tract infections. Proceedings of the Annual Scientific Congress of Sri Lanka College of Paediatricians. 1997 July 16-20; Colombo, Sri Lanka.

28. David B S. Childhood urinary infection. Transactions of the Ceylon College of Physicians. Sept 1971; 4: 29-47. 
29. Gunaratna I N A, Hettiarachchi P S H, Jayasekera K P R. Radiological investigation for vesicoureteric reflux and renal scarring. Proceedings of the Annual Scientific Congress of Sri Lanka College of Paediatricians. 1997 July 16-20; Colombo, Sri Lanka.

30. Smellie J M, Prescod N P, Shaw P J, Risdon R A, Bryant T N. Childhood reflux and urinary infection: a follow-up of 10-41 years in 226 adults. Pediatr Nephrol 1998; 12:727-73.

31. Jacobson S H, Eklof O, Eriksson C G, et al. Development of hypertension and uraemia after pyelonephritis in childhood: 27 year follow up. British Medical Journal 1989; 299: 703-6.
32. Indian Paediatric Nephrology Group, Indian Academy of Paediatrics. Consensus statement on management of urinary tract infections. Proceedings of the XIII National Conference of Paediatric Nephrology. 2001 Nov 17-18; Calcutta, India.

33. Stark H. Urinary tract infections in girls: the cost effectiveness of currently recommended investigative routines, Pediatric Nephrol 1997; 11:174-7. 
